avaient été ainsi cédés en toute propriété, sans aucune réticence ni réserve de droits d'usage ou autres,

Mille hectares, la première tentative sérieuse de reboisement, c'était une contenance, une acquisition des plus précieuses. Tous les patriotes qui s'intéressent à ces questions vitales avaient les yeux fixés sur ces terrains réparateurs dont ils escomptaient d'avance la renaissance prochaine.

En effet, le service forestier se mit en devoir d'aménager le sol, à chers deniers, aux frais de l'Etat et de faire de coûteux repeuplements. La restauration marchait à souhait. Les semis manifestaient les plus belles espérances d'avenir. Encore quelques années et de magnifiques forêts allaient recouvrir ces lieux hier encore nus et arides.

Mais c'était bien mal connaître l'esprit des pasteurs voisins. Ils oublièrent qu'ils avaient vendu ces terres et dès qu'ils virent de la verdure, ils ne songèrent plus qu'à la faire manger. Quelle bonne aubaine! C'était là du lait et de la viande pour rıen; rien que la peine de les prendre.

Ils s'empressèrent d'y mettre leurs troupeaux dont chaque coup de dent détruisait un arbre.

Le petit jeu des procès-verbaux que je vous ai expliqué tout à l'heure recommença, avec son succès habituel.

Impatienté, le service forestier voulut pour une fois, user de l'autorité qui lui est donnée par la loi et faire enfin respecter la chose publique, la propriété dont il a la garde.

Approuvé par un préfet d'un esprit droit, il allait saisir les troupeaux de ces déprédateurs obstinés, et les vendre au profit du Trésor, ainsi que le Code forestier lui en faisait justement un devoir, lorsqu'une intervention électorale s'étant produite, il reçut ordre de laisser faire. Il paraît que l'Administration en saisissant quelques moutons et quelques vaches, pour donner un salutaire exemple à toutes les Pyrénées, exemple qui eut été décisif, 'allait mettre tout le pays à feu et à sang.

Ces exagérations et ces défaillances sont lamentables!

Aujourd'hui cette espérance d'avenir est détruite par l'influence néfaste que je viens de vous signaler.

Eh! bien, il faut que ces faits soient connus, de telle sorte que le suffrage universel de la plaine se révolte enfin contre ces scandaleux abus du suffrage universel de la montagne.

Car, qu'on ne s'y trompe pas, il n'y a pas de politique dans cette affaire, mais seulement la satisfaction des plus misérables intérêts personnels, s'exerçant au détriment de la chose publique, de la sécurité, de la vie et de la propriété des électeurs de la plaine.

En conséquence des différents travaux produits, les vœux suivants ont été adoptés à l'unanimité par le Congrès :

\section{RÉFORMES LÉGISLATIVES}

$1^{0}$ Soumission au régime forestier de tous les terrains communaux plus ou moins boisés intéressant le régime des caux, que ce soit en montage, en plateau ou en cotean.

$2^{\circ}$ Complément du titre XV du Code forestier de manière à empêcher tout défrichement illicite, même indirect.

3o Soumission à un régime pastoral des pelouses, vacants et pâturages à jouissance collective, intéressant également le régime des eaux.

$4^{\circ}$ Abandon de l'interprétation étroite donnée jusqu'ici à la loi du 4 avril I 882 , ou la modification de cette loi en vue de la création de périmètres de régularisation du régime des eaux au même titre que les périmètres de correction des torrents, assimilant les faits de dénudation à ceux d'érosion.

50 Extension des dispositions de l'article $V$ de la loi du 4 avril 1882 , pour permettre dallouer des subventions en nature, des primes en argent ou en travaux, aux travaux de reboisement exécutés dans les régions de coteaux où l'influence de ces mêmes travaux sur la régularisation du régime des eaux est manifeste.

$$
\begin{gathered}
\text { GUÉNOT, } \\
\text { Secrétaire général adjoint du Congrès du S.-O.N, }
\end{gathered}
$$

\section{LÉLECTROCHINIE SUR LES BORDS DU NIAGARA}

J.-W. Richards, à l'occasion du second meeting de l'American electrochemical Society, saluait les rivages du Niagara comme le " home par excellence » de l'industrie électrochimique américaine et, de fait, si cette industric possède sur le territoire de l'Union d'autres centres, autour desquels sont rassemblées les usines électrochimiques en plus ou moins grand nombre, nulle part on ne peut voir réuni sur un même point un groupe aussi puissant d'entre. prises d'utilisation chimique de l'énergie hydro-électrique.

Toutes les applications de l'électricité à la chimie n'y sont cependant pas représentées; c'est ainsi qu'il ne s'y trouve aucun de ces établissements de raffinage électro. métallurgique, si prospères ailleurs, en Amérique; mais on y rencontre quelque représentant de chacun des genres d'industrie qui exigent pour la transformation de leurs produits, des quantités considérables d'énergie et, qui, manipulant et livrant des matières de valeur relativement basse, ne peuvent subsister qu'en disposant de sources très économiques de la puissance motrice et de moyens commodes et peu coûteux de transport. A cet égard, il n'est pas de situation plus privilégiée que celle de Niagara Falls City, au centre des grands lacs, au milieu du systeme de canaux et de voies fluviables qui en rayonnent et amènent le charbon demandé par les opérations accessoires au pris de un dollar et demi, au pied de chutes, enfin, qui recèlent sous une forme d'utilisation facile, plus de sept millions de chevaux.

Sur un terrain si éminemment favorable à leur activité, les électro-chimistes ont, depuis longtemps, remporté des succès brillants et répétés, qui ont attiré sur cette ville l'attention soutenue de la presse technique et du monde scientifique; aussi est-il facile, d'après les monographies, les descriptions de fabriques, les études de procédés ou de méthodes de travail, parues de tous côtés, de se créer une idée du développement qu'y a pris l'électro-chimie.

Un caractère commun des établissements électrochlmiques du Niagara est de ne point produire eux-mêmes l'énergie électrique qu'ils consomment, et de la demander à deux stations formidables de distribution de force quif puisent leur puissance dans les chutes et la leur distribuent avec les avantages qui découlent de la centralisation indus trielle. La description de ces stations centrales sort du cadre de cette étude; il suffira d'indiquer que, dans leur état présent, elles peuvent fournir sous 2200 ol 2500 volts de tension 60000 chevaux; qu'elles achèvent, en ce moment, l'aménagement de 50000 , et commencent celui de 100000 autres chevaux; enfin, qu'elles possèdent une réserve de concessions de 250000 chevaux encore. Sur ce nombre, l'électrochimie en absorbe à elle seule 40000 , á 
des tarifs qui, en raison du mode de travail continu et égal de cette clientèle, peuvent s'abaisser en certains cas jusqu'au taux de 18 et même 14 dollars par an. Les consommateurs transforment le courant à leur convenance, le plus souvent en continu, par des génératrices qu'entraînent des moteurs alternatifs.

Quelques-uns des procédês exploités dans ces conditions sont connus en Europe, soit qu'ils y soient nés, et aient été transportés ensuite, peu modifiés, sur le territoire américain, comme c'est le cas pour le procédé de Castner, soit que les découvertes sur lesquelles ils se basent, et les essais qu1 les ont suivies, se soient produits simultanément sur les deux continents : ainsi le procédé Hall, si voisin et contemporain du procédé Héroult. Mais d'autres ont une forte marque d'originalité, et l'ancien monde ne possède encore rien d'équivalent aux procédés de Salom, de l'Atmospheric products Cy et de l'United Baryum Cy.

La plus ancienne installation est celle de la a Pittsburg reduction Co » qui prépare depuis I 888 l'aluminium à l'aide du procédé Hall, et, par le nombre des chevaux qu'elle emploie, est au premier rang des usines électro-chimiques du Niagara.

Elle nous fournit le type des électrolyses industrielles à l'état de fusion ignée, et il semble logique d'examiner à sa suite les fabriques utilisant comme elle l'électrolyse, soit des corps fondus, soit des corps dissous, en réservant pour plus tard la description des procédés, qui n'emploient la puissance électrique que comme source d'énergie calorifique.

\section{I. - ELEGTROLYSE DE SELS FONDUS}

Par les deux usines qu'elle possède au Niagara, "THE Pirtsburg Reduction Co » est la productrice d'aluminium la plus importante du monde entier. Les câbles d'aluminium de r boo mètres, qui relient l'une d'elles à l'une des stations hydro-électriques, lui apportent 6000 chevaux, sous 2200 volts alternatifs; elle les reçoit dans des transformateurs qui en rabaissent la tension à $\mathrm{r} 30$ volts, et redresse ensuite le courant en continu de 160 volts dans des convertisseurs rotatifs de 600 kilowatts. L'autre, fondée depuis plus de 10 ans au voisinage (60o mètres) de la seconde station hydro-électrique, lui emprunte actuellement 9000 chevaux en courant continu, issu de dynamos Westinghouse de 750 chevaux, calées directement sur les turbines; le voltage d'utilisation est ici de 280 volts.

La méthode de travail usitée - celle de Hall - est assez analogue à celle de Héroult dans son principe : l'alumine, dissoute dans la cryolithe et le fluorure d'aluminium fondus, est décomposée par le courant, en métal qu'on recueille et oxygène qui disparaît sous forme de gaz carbonique, après avoir brûlé le charbon des anodes; elle est continue : les électrolyseurs supportent cinq à six mois de marche ininterrompue, en fournissant chaque jour une coulée de métal.

Leur construction est simplifiée le plus possible. Ce sont de grandes caisses de fer, allongées, à double fond: dans celui-ci, haut de io $\mathrm{cm}$., est glissé du charbon de bois afin de réduire les pertes par conductibilité; par dessus est tassée une pâte de goudron, de coke pulvérisé et de débris d'anciennes électrodes, en une couche épaisse de
$40 \mathrm{~cm}$. d'un côté et de 50 de l'autre: la surface en est, de la sorte, inclinée dans le sens de la longueur de la caisse vers un trou de coulée qui perce une des faces extrêmes de celle-ci ; les parois latérales sont garnies d'un enduit analogue de $20 \mathrm{~cm}$. d'épaisseur. Le tout est ensuite porté au rouge pendant une vingtaine d'heures dans l'un des fours spéciaux que l'on a réunis en batterie dans un bâtiment de l'usine abritant les services accessoires, tels que les ateliers d'adaptation des électrodes. On en retire une auge rectangulaire de coke, homogène, blindée de fer ; les dimensions intérieures en sont approximativement $1 \mathrm{~m}$. 40 sur o m. 6o, dans le sens horizontal, avec o m. 40 de profondeur vers un bout et $\mathrm{o} \mathrm{m}$. 50 à l'autre.

C'est là que l'on fond le mélange de cryolithe, de fluorure d'aluminium et d'alumine, quelquefois additionné encore de spath fluor, qui constitue l'électrolyte. Dans ce bain, on enfonce de 15 à $25 \mathrm{~cm}$. l'extrémité de forts cylindres de charbon ayant $50 \mathrm{~cm}$. de larg. et $3 \mathrm{~cm} .75$ de diamètre, de manière qu'ils atteignent jusqu'à $3 \mathrm{~cm}$. du fond: on en dispose quarante par auge sur quatre rangées. Leur partie supérieure porte un axe de cuivre, prolongé jusqu'au conducteur positif de la canalisation électrique; il sert à établir le contact et à la manœuvre de ces charbons. Les parois de fer sont reliées à l'autre pôle, et, par suite, le coke du revêtement intérieur et de la sole sert de cathode. L'électrolyse s'effectue avec 10000 ampères sous un peu moins de 5,5 volts; le réglage se fait en agissant séparément sur chacune des anodes. Grâce à ces hautes intensités, la température de la masse fondue se maintient à peine au-dessous de $900^{\circ}$, sans aucun chauffage extérieur.

Pour éviter les pertes par radiation et volatilisation, pour supprimer aussi l'action de l'air sur le bain et les électrodes, on achève de remplir la caisse avec du poussier de charbon de bois. L'alimentation en alumine neuve et les quelques additions de cryolithe qui sont nécessaires pour compenser les pertes, se font par projection de ces matières sur cette couche protectrice; pendant qu'elles la traversent elles ont tout le temps de perdre leur humidité et arrivent sèches dans le bain; on maintient libres la surface et les bords de celui-ci, en brisant avec des ringards les croûtes qui tendent à s'y former.

A la mise en marche d'un four, et pendant la première période de son fonctionnement, tant que la cryolithe mêlée au bain n'est pas entièrement dépouillée de son fer et de son silicium, on attache peu d'importance à la pureté de l'alumine introduite; ces éléments étrangers se retrouvent dans le métal obtenu qui n'est alors propre qu'aux usages de la métallurgie. Mais ensuite on s'adresse à de l'alumine soigneusement purifiée, d'importation allemande ; impure elle se prêterait mal à l'électrolyse continue et en rendrait la conduite délicate; le métal qu'on en tire est fort pur et ne contient guère que $3 \%$ de silicium et de a à $5 \%$ de fer (I).

La Compagnie a cherché à se libérer du tribut qu'elle paie de ce chef à l'étranger, en mettant sur pied deux procédés de purification encore en essais : l'un, dû à Hall, est

(1) Ce métal est refondu et ouvré dans une troisième usine que possède la Société; il se vend x fr. 55 . 
une fusion de l'alumine brute au four électrique, avec une petite quantité de coke qui élimine les impuretés sous forme de ferrosilicium plus ou moins titanifère; l'autre est un traitement par voie humide, de la bauxite par la chaux.

Les électrolyseurs sont disposés en série, de manière à utiliser toute la tension donnée par les génératrices; chacun d'eux fournit par jour 50 à 55 kilogrammes d'aluminium en lingots de ro kilogrammes, et absorbe 65 chevaux : la fabrication de un kilogramme de métal exige donc la dépense d'environ 3o chevaux-heure. L'utilisation du courant n'est guère que de 70 à $75 \%$ et varie assez notablement en raison de la facilité des courts circuits, et d'une redissolution partielle dans le bain, du métal libéré, par un phénomène encore obscur, mais souvent constaté déjà sur d'autres électrolytes fondus.

Malgré cela, et malgré l'obligation d'une purification onéreuse de l'alumine, "The Pittsburg Reduction Co " lutte victorieusement arec les producteurs de l'aluminium et de ses alliages par les procédés électrothermiques, qui, pour le même résultat, consomment le double d'énergie. L'aménagement prochain par cette Société, à Massena et à Schawinigan Falls, de 17000 chevaux pour la métallurgie de l'aluminium, est un signe certain de sa prospérité.

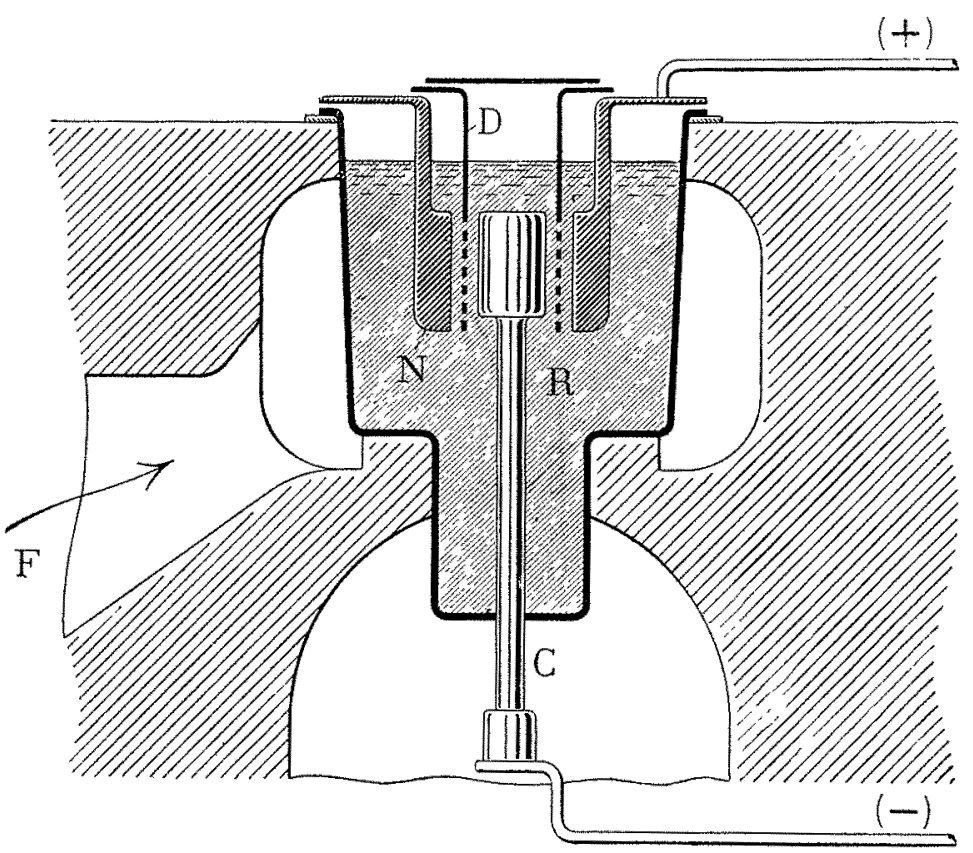

Fig. I

“The Niagara electrochemical Co » pareillement, prépare un métal, le sodium, par électrolyse d'un de ses composés fondu, la soude caustique, suivant les indications de Castner. L'opération se fait à une température aussi voisine que possible du point de fusion de l'électrolyte qui, pratiquement, est maintenu au-dessous de 3 r $50-320^{\circ}$; une élévation du bain de quelques degrés au-dessus de cette limite augmenterait considérablement son pouvoir dissolvant pour le métal libre qui, diffusant alors dans la masse, se recombinerait à l'anode avec l'oxygène.

On place la soude dans un récipient légèrement tronconique de fer $\mathrm{R}$ (fig. I), ayant $45 \mathrm{~cm}$ de diamètre moyen, et 6 o centimètres de hauteur, chauffé sur le côté par les gaz d'un foyer $\mathrm{F}$. Le fond est prolongé par un cylindre plus étroit, en fer également, de 30 centimètres de long. et refroidi à l'air extérieur ; c'est par là, que la cathode est introduite ; l'alcali s'y solidifie en formant joint étanche entre elle et le fer des parois. Cette électrode en charbon C s'élève jusqu'au milieu du récipient, oủ elle s'élargit et atteint un diamètre de $10 \mathrm{~cm}$; concentriquement, et à la même hauteur, est suspendu un cylindre de nickel $\mathrm{N}$, de $18 \mathrm{~cm}$ de diamètre, qui joue le rôle d'anode; entre deux, pour éviter le con. tact des globules du métal séparé sur le charbon, avec l'anode, est tendue sur une hauteur de $15 \mathrm{~cm}$, une toile de nickel qui se prolonge vers le haut par un cylindre de nickel de mêmes dimensions $\mathrm{D}$; celui-ci dépasse un peu la surface de la soude fondue; un couvercle de tôle en met l'intérieur à l'abri de l'air, sans empêcher le départ de l'hydrogène; c'est dans cet espace que le sodium fondu plus léger que son hydrate, se rassemble; un déversoir permet de le couler dès qu'il est assez abondant. L'oxygène mis en liberté à l'anode est perdu.

Une cuve de cette grandeur reçoit une charge de 115 kil. de soude ; elle est traversée par 1 200 ampères sous 5 volts, ce qui correspond à des densités de courant de 17 ampères par dmq d'anode et 32 ampères 5 par dmq de cathode. Au début de l'électrolyse, l'eau, introduite par l'alcali à l'état d'humidité, est seule décomposée, et de l'hydrogène se dégage; dès qu'elle est éliminée totalement, le sodium appa* raît, et le rendement se maintient à $90 \%$ si le chauffage est bien réglé.

Cette usine possède I 20 électrolyseurs disposés en 4 séries de 30 . Ils livrent 2800 kilogr. de métal par jour moyennant une dépense de 1000 chevaux; chaque kilogr, de. mande 9 chevaux-heure et revient à ${ }_{1}{ }^{f}{ }_{1} 5$ au plus.

Une partie de la production est écoulée sous la forme métallique après une nouvelle fusion; la plus grande portion est convertie en divers dérivés du sodium : en cyanure, par chauffage avec des matières organiques azotées; sur* tout en bioxyde, par oxydation au rouge, dans un tube de fer traversé par un courant d'air; ce bioxyde està 95-97\%, le surplus étant du carbonate, et se vend 80 cent. le kilogr. Malgré la demande limitée du marché pour ces produits, la situation a paru assez favorable à la Société pour qu'elle soit en voie d'augmenter ses installations.

Dans son allure générale le procédé exploité par "THe ACKER PROCESS Co » présente quelque analogie avec le précédent; le but en est la production du chlore et d'un alliage du sodium - le plomb-sodium, - par l'électrolyse du sel marin fondu; sous l'action de la vapeur d'eau surchauffée, l'alliage donne ensuite de la soude. Des essais en grand, basés sur le même principe ont été effectués, il y a quelques années, dans les Alpes françaises, mais le succès n'a pas couronné les efforts très ingénieux de nos inventeurs, tandis que, déjà, l'usine américaine où en a été réalisée l'application, est depuis deux ans et demi en pleine marche, avec 3250 chevaux, et répond, pour une grosse part, àla demande des consommateurs d'outre-mer, à qui elle offe plus de 11 tonnes de soude et de 10 tonnes de chlore par jour.

Les difficultés qui ont fait échouer les prédécesseurs de Acker ont été supprimées par lui, en n'introduisant dans son électrolyseur que du chlorure de sodium complètement desséché, ce qui évite la formation d'acide chlorhydrique par le chlore au rouge et l'eau; en supprimant tout 
chauffage extérieur et demandant la chaleur nécessaire à la fusion au courant électrique lui-même; en faisant circuler très vivement le plomb, qui emprisonne l'alliage formé et l'empêche de revenir à la surface; en adoptant le graphite comme matière des anodes; en décomposant le plomb sodium fondu, à l'état très divisé, par de la vapeur d'eau sous pression.

Dans une cuve rectangulaire en fonte $F$ (fig. 2 ), ayant $\mathrm{I}^{\mathrm{m}} 35$ sur $0^{\mathrm{m}} 55$, munie d'un double fond, et revêtue intérieurement de brique ordinaire sur les parois latérales, sont disposées une première couche de plomb fondu $\mathrm{P}$, recouvrant de 4 centimètres le fond supérieur, et par dessus une seconde couche de chlorure de sodium fondu $\mathrm{S}$ de 20 centimètres d'épaisseur; dans celui-ci plongent jusqu'à 2 cent. 5 de la surface du plomb, quatre blocs de graphite d'Acheson A, de section rectangulaire, ayant $19 \mathrm{~cm}$. sur 35 de côté : ce

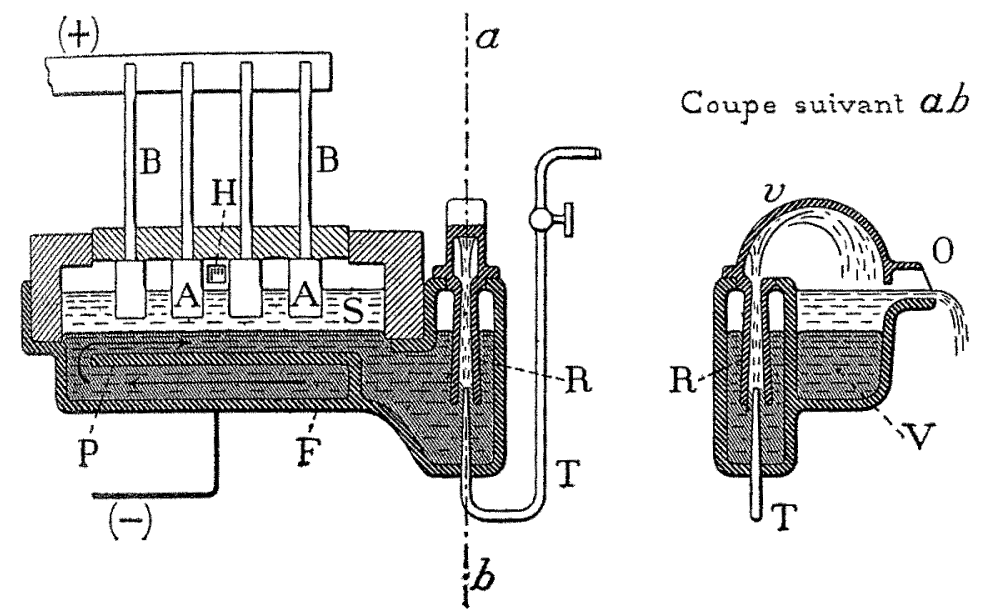

Fig. 2

sont les anodes reliées, à travers le couvercle en briques, à la canalisation électrique, par des bâtons de charbon plus mince $\mathrm{B}$; le bac de fonte, et, par son intermédiaire, le plomb, est relié au pôle négatif. Entre les électrodes passent 8000 ampères, moyennant une différence de potentiel de 7 volts; ces hautes densités de courant, de près de 300 ampères par décimètre carré d'anode, sont nécessaires pour maintenir la fusion de l'électrolyte, mais forcent à travailler sous une tension électrique élevée, et conduisent ainsi à un médiocre rendement en énergie. Le chlore est appelé, par une ouverture latérale $\mathrm{H}$, dans les chambres à chlorure de chaux, au moyen d'un aspirateur; sous l'action de ce dernier une légère dépression règne dans l'atmosphère de l'électrolyseur, et des rentrées d'air se produisent par les joints, mais elles ne présentent pas d'inconvénient pour l'utilisation du gaz recueilli. Le plomb enrichi en sodium s'écoule en passant sous une cloison en briq'xe, dans une protubérance $R$, de la cuve de fonte, où pénètre un tube étroit $\mathrm{T}$, amenant de la vapeur d'eau sous la pression de 2,8 atmosphères. Ce tube débouche au bas d'un cylindre vertical en fer de 4 cent. 5 de diamètre; le jet de vapeur entraine le plomb par celui-ci et le projette violemment contre le toit voûté $\nu$ d'une petite chambre latérale $\mathrm{V}$, servant à la décantation des produits de la réaction. Ce dispositif divise très finement l€ plomb-sodium, qui se dépouille en fort peu de temps de son métal alcalin, en dégageant de thydrogène et donnant de la soude fondue; ces produits se séparent facilement, dans la chambre de décantation; le plomb tombe dans le fond; la soude s'écoule par un déversoir $\mathrm{O}$ percé dans une des parois au-dessus du niveau du plomb et l'hydrogène s'échappe. La soude est recueillie, fondue une seconde fois, de manière à en séparer les impuretés plus denses qu'elle, et livrée au commerce ; le plomb revient par le double fond à l'autre extrémité de l'électrolyseur; l'injection de vapeur suffit à assurer la continuité de son mouvement.

Le sel est introduit brut dans la cuve de fonte par une ouverture du couvercle, après avoir séjourné quelque temps sur celui-ci et y avoir perdu son humidité.

Les 45 fours de ce modèle, actuellement en service, absorbent chacun 75 chevaux, et livrent par jour, chacun 26 . kilogs de soude à $97,7 \%$; leur rendement en quantité est remarquable et peut atteindre jusqu'à $93 \%$. Cette solution élégante d'un problème, où plusieurs ont échoué. présente un point faible; c'est le voltage sous lequel se fait l'électrolyse, en raison duquel le cheval électrique ne produit par jour que $3,45 \mathrm{kgr}$. de soude et $3, \mathrm{rgr}$. de chlore.

\section{ELECTROLYSE DE GORPS DISSOUS}

De fondation beaucoup moins récente, presque aussi ancienne que la "Pittsburg " et "l'Electrochemical " est la "Castner electrolytic Alkali Co ", autre fabrique de chlore et de soude caustique; à la différence de la précédente elle opère sur du sel dissous. Située à moins de I. 500 mètres des usines productrices elle en reçoit 6.000 chevaux en courant alternatif de 2500 volts, et le transforme en continu de 230 volts par des transformateurs de 200 chevaux reliés à des commutatrices rotatives.

Le procédé exploité dans cette installation est celui de Castner-Kellner, mis au point par Mauran. Il consiste à électrolyser, au-dessous de $50^{\circ}$, une solution concentrée de sel marin, en employant une électrode intermédiaire en mercure. Celle-ci, dans une première partie de l'appareil contenant le sel, cst placée en cathode, et amalgame le sodium libéré par le courant à sa surface, tandis que le chlore se dégage à l'anode; dans une seconde partie cet amalgame est disposé en anode au contact d'eau pure et lui cède son sodium sous forme de soude, pendant que l'hydrogène se sépare à la cathode.

Théoriquement, ces deux opérations nécessitent la même quantité d'électricité; mais en raison du rendement plus faible de la première, il ne faut admettre, pour effectuer la seconde que $90 \%$ seulement du courant total qui traverse l'électrolyseur, sinon le mercure est attaqué.

L'électrolyseur représenté schématiquement ci-après est formé par une caisse à trois compartiments (fig. 3), entièrement construite en plaques d'ardoise jointes avec un lut de caoutchouc; ses dimensions sont I $\mathrm{m}$. 40 en large et en long et $15 \mathrm{~cm}$ en hauteur; il reçoit $45 \mathrm{kilog}$. de mercure et roo litres de solution salée. Les cloisons intermédiaires ne joignent pas le fonds et laissent au-dessus de celui-ci un vide de o m. i 5 augmenté, du reste, par une rainure $r$ creusée en regard, dispositif qui permet d'un côté à l'autre la circulation du mercure; celui-ci s'étend sur le fond en une couche $m$ de 'o,2 $\mathrm{cm}$ d'épaisseur. La saumure provenant de la dissolution du sel marin brut est 
placée dans les compartiments extrêmes où plongent les anodes A au nombre de 12 , et circule dans la série des appareils par l'action d'une pomve en grès; elle repasse sur du sel neuf lorsqu'elle est trop appauvrie. Les anodes sont des blocs allongés de graphite à section de rail: la tête repose au-dessus du couvercle ; un lut très soigné au ciment rend leur assemblage étanche, le patin arrive à I cent, 5 du mercure; elles sont toutes reliées en dérivation par des lames de plomb paraffiné. C'est là que se forme l'amalgame de sodium à $1 / 1000$ qu'on détruira dans la chambre voisine. Le chlore est évacué à l'aide de tubes en caoutchouc dans une canalisation en plomb reliée à un aspirateur et aux chambres d'absorption.

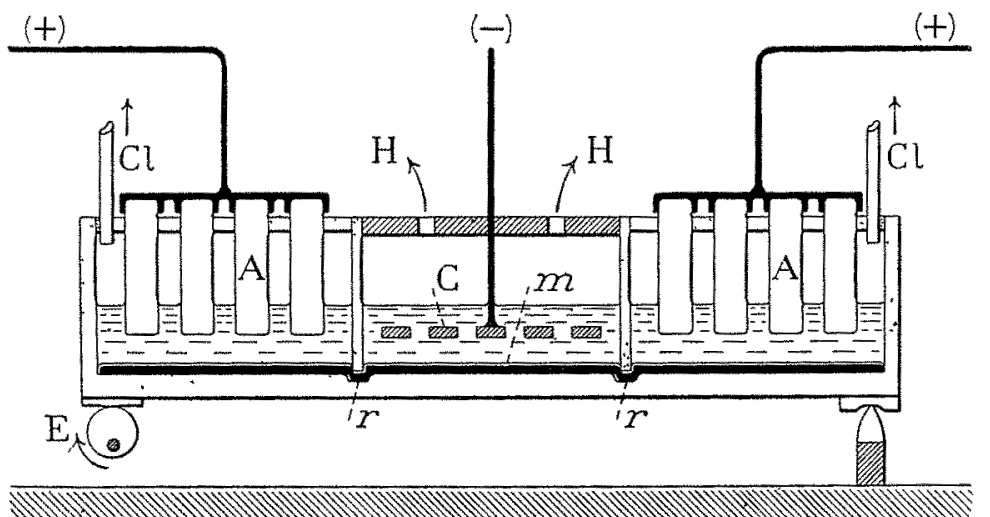

Fig. 3

Le compartiment central reçoit de l'eau pure; il contient la cathode; cette électrode $\mathrm{C}$ est formée d'une grille de fer suspendue horizontalement à $2 \mathrm{~cm}$ du fond; les barreaux, carrés, mesurent $2 \mathrm{~cm} 5$ d'épaisseur et laissènt entre eux des vides de $\mathrm{I} \mathrm{cm} 5$. L'amalgame s'y décompose sous l'influence du courant en donnant de la soude caustique. Lorsque la lessive alcaline qui s'y forme ainsi atteint $3 \mathrm{I}^{\circ}$. Baumé, on l'en extrait et la concentre dans des chaudières de fer.

Le mouvement du mercure est obtenu en élevant et abaissant une fois par minute, par un excentrique $\mathrm{E}$, l'une des extrêmités de la caisse, l'autre reposant sur un pivot fixe. Le mercure passe ainsi successivement dans les trois parties de l'électrolyseur, amalgame le sodium aux extrémités de sa course et le cède à l'eau en traversant la chambre centrale (I).

Chacun de ces appareils absorbe 630 ampères sous 4 volts 3 , les densités de courant admises étant de 9 amp. au décimètre à la cathode de mercure et 12 à l'anode. Avec ses 540 électrolyseurs disposés en dix séries de 54 , donnant un rendement moyen de $90 \%$ en quantité, l'usine livre par jour 85 tonnes de chlorure de chaux à $36 \%$ de chlore et 34 tonnes de soude à $98 \%$. Le matériel a une résistance suffisante pour ne point grever beaucoup le prix de ces produits ; les électrolyseurs, les cathodes, le mercure même, si le compartiment central est assez shunté, ne s'usent nullement; seules les anodes doivent être remplacées au bout de deux à trois années de service.

(1) Dans les derniers modèles de ces électrolyseurs, le pivot est au centre de la caisse. Par cette disposition leur mouvement se fait avec une dépense infime de travail.
La faiblesse relative du voltage nécessaire est un gros avantage pour ce procédé par comparaison à celui deAcker; mais l'obligation de concentrer des lessives étendues, un moins bon rendement en quantité, l'amortissement onéreux du capital mercure, le contrebalancent assez pour que les deux méthodes concurrentes puissent vivre côte à côte.

A 3 kilomètres des chutes, d'une importance beaucoup plus modeste, bien que poursuivant un but analogue, est l'établissement de. "The Roberts Chemical Co », qui, avec 500 chevaux seulement, prépare de la potasse caustique et de l'acide chlorhydrique, par électrolyse du chlorure de potas. sium dissous.

L'électrolyseur en usage est du type à diaphragme (bre. vets Roberts) (fig. 4). Un cylindre en tôle hermétiquement fermé $\mathrm{C}$ reçoit de la lessive de potasse faible; sa surface interne forme la cathode; il contient un cylindre de même hauteur mais plus étroit $\mathrm{P}$, en porcelaine poreuse, imprégnée de silice gélatineuse, tout rempli de grains d'un charbon peu conducteur; ceux-ci, fortement serrés par les bouchons isolants B qui ferment les extrémités, maintiennent, suivant l'axe, l'anode constituée par un bâton de charbon conducteur A. Pour éviter l'attaque de la silice par l'alcali, un manchon $M$, de porcelaine poreuse ordinaite, entoure ce cylındre, et, dans l'espace annulaire qui l'en sépare, sont glissés des fragments de porcelaine destinés à empêcher les mouvements de convexion du liquide.

Des tubes permettent de faire circuler dans le cylindre de tôle la potasse caustique, dans le cylindre poreux du chlorure de potassium saturé; ces solutions passent successivement dans une série de ces appareils. D'autres tubes recueillent l'hydrogène dégagé sur là cathode, le chlore mis en liberté à l'anode, et ces gaz se recombinent ensuite, dans un brûleur spécial, en gaz chlorhydrique, que l'on condense dans l'eau d'un appareil d'absorption.

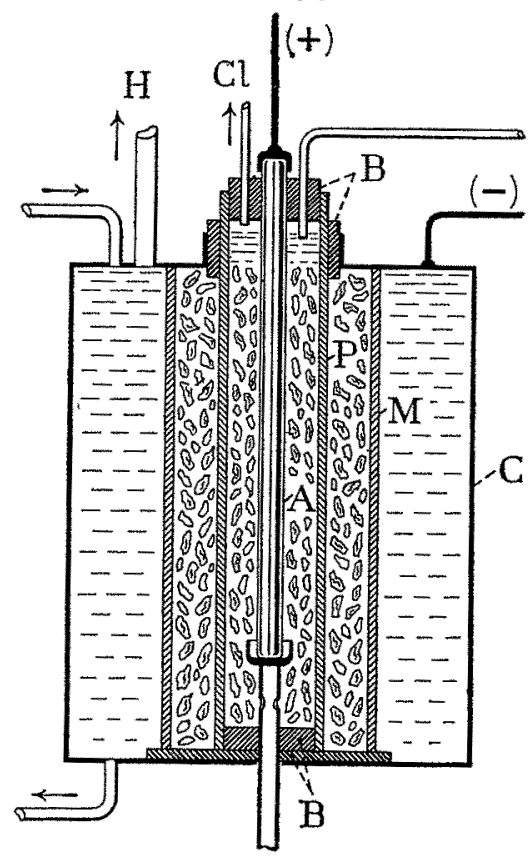

Fig. 4

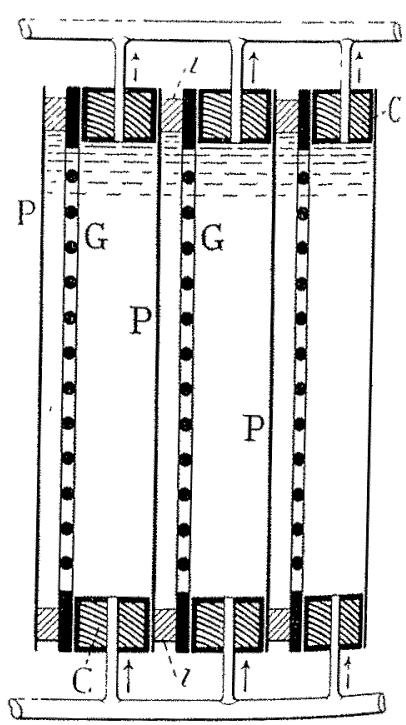

Fig. 5
Des procédés analogues donnent ailleurs de bons résultats; mais ici, les difficultés de mise au point qu'offrent les électrolyseurs à diaphragme, la perte en acide, nécessaire pour conserver une faible acidité au liquide qui baigne l'anode, l'exiguité des moyens d'action, dans une industrie 
oú l'ampleur est une condition de réussite, gênent le fonctionnement de cette Société qui paraît ne donner qu'une production intermittente.

La transformation des chlorures alcalins occupe encore deux autres fabriques au Niagara: "The National electrolytic Co », "The Oldbury Chemical Co », qui oxydent électrolytiquement le chlorure de potassium en chlorate.

La première, l'une des plus anciennes usines de ce genre, y arrive au moyen des méthodes un peu vieillies de Gibbs et Franchot, par l'électrolyse sans diaphragme d'une solution neutre de chlorure (fig.5). Cette opération se fait dans un bac constitué par une série de cadres rectangulaires $\mathrm{C}$, de bo $\mathrm{cm}$ sur $60 \mathrm{~cm}$ environ, assemblés à la manière des cadres d'un filtre-presse ; ils sont en bois de $5 \mathrm{~cm}$ d'épaisseur, recouvert d'une feuille de plomb; contre une des faces est appliquée l'anode $\mathrm{P}$, mince lame de platine; contre l'autre, la cathode $\mathrm{G}$, sorte de grillage léger en cuivre muni de renforcements diagonaux; entre deux cadres semblables, au moment de l'assemblage, on interpose un cadre en caoutchouc $i$ de mêmes dimensions latérales qui les maintient éloignés de $1 / 2 \mathrm{~cm}$; quelques baguettes de verre préviennent tout contact du cuivre de l'un d'eux avec le platine de l'autre. Deux anodes successives limitent ainsi une sorte de cellule indépendante où circule du chlorure à $30 \%$ à raison de 54 litres par heure; le courant électrique y pénètre par l'électrode en platine, traverse l'électrolyte pour gagner l'électrode de cuivre, et, par le plomb du cadre, atteint la seconde feuille de platine, d'où il pénètre dans la cellule voisine. L'ampérage admis est élevé : 56 ampères par décimètre, condition qui atténue la réduction cathodique, mais occasionne des pertes par effet Joule: la solution qui entre à la température ordinaire, lorsqu'elle s'écoule, marque $55^{\circ}$ à $65^{\circ}$; elle abandonne son chlorate par refroidissement. Le rendement électrique s'élève à 68 et $70 \%$ en quantité, résultat assez favorable; mais, par suite des pertes, un cheval-jour ne donne guère que 2 kilogrammes de chlorate et la production des 2.000 chevaux absorbés par cette fabrication est relativement faible.

Cette puissance arrive à l'usine en triphasé par un transport de faible longueur $(175 \mathrm{~m}$.) ; des commutatrices redressent le courant en continu avant son emploi.

Les conditions de préparation paraissent de beaucoup meilleures à l" "Oldbury Chemical. Co » qui, avec 700 chevaux seulement, livre par jour goo kilogrammes de chlorate de potasse et 450 de phosphore ordinaire; mais les opérations de cette Compagnie sont entourés d'un tel secret, qu'il est impossible de se procurer quelque renseignement sur son mode de travail, surtout en ce qui concerne le chlorate. Quant à la préparation du phosphore, malgré les nuages dont elle est environnée, on en connaît du moins le prıncipe, et quelques données qui seront exposées plus loin avec d'autres exemples d'utilisation électrothermique du courant en chimie.

Avant d'abandonner celles des usines tributaires du Niagara, dont le fonctionnement est basé sur l'emploi de l'électrolyse, il faut en citer une qui a inauguré, en électrométallurgie, une méthode toute nouvelle, et qui, fort jeune encore et de faible importance, paraît cependant déjà devoir faire école. C'est la fonderie de plomb de « The electricai. Lead Reduction Co ", qui, avec 5 no chevaux, extrait de la galène riche, par le procédé Salom, un métal assez pur pour recevoir des utilisations immédiates (fig. 6).

Le minerai, très finement pulvérisé, enrichi par un traitement magnétique jusqu'au titre de $83 \%$ o de métal, est étendu sous une épaisseur $m$ de 2 cent. 5 dans le fond de larges cuvettes $\mathrm{C}$ en plomb antimonié, ayant $38 \mathrm{~cm}$ de diamètre au fond et $46 \mathrm{~cm}$ au bord supérieur, avec I $5 \mathrm{~cm}$ de profondeur; on empile plusieurs de ces cuvettes (douze), les unes sur les autres, en les séparant par des

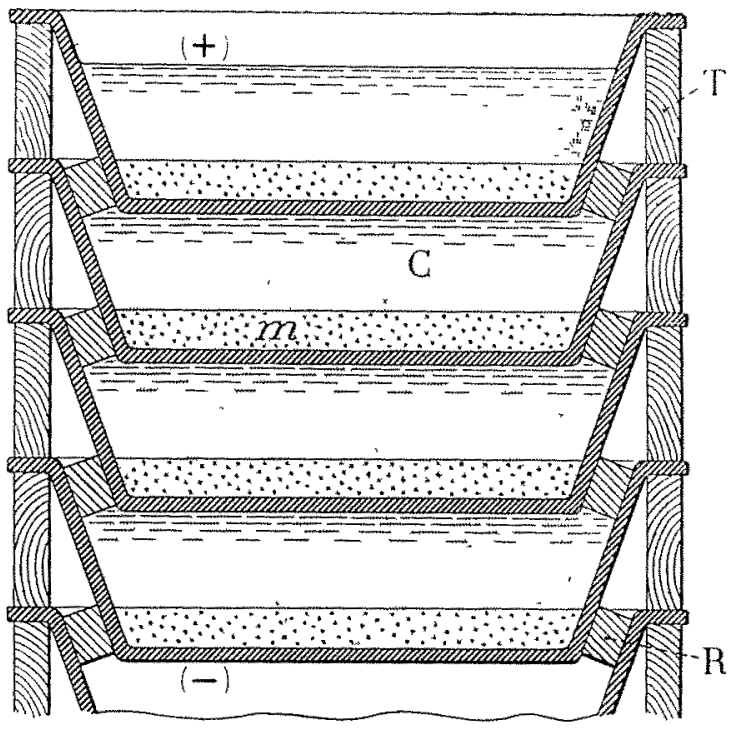

Fig. 6

rondelles de caoutchouc $\mathrm{R}$, et des taquets isolants $\mathrm{T}$; on garnit ensuite l'espace vide entre elles d'acide sulfurique à ro o/o. En reliant la cuvette la plus élevée, qu'on ne remplit point, au pôle positif de la distribution électrique, et la cuvette inférieure au pôle négatif, le courant passe de l'une à l'autre, en traversant l'acide et la galène, sur toute la hauteur de la pile; le fond de chaque cuvette, sur sa face interne, couverte de galène, sert de cathode, et sur sa face externe sert d'anode pour sa voisine inférieure. Par l'électrolyse, de l'hydrogène se dégage sur le minerai, lui enlève le soufre qu'il contient sous forme d'hydrogène sulfuré et laisse le plomb. C'est une application très originale et inattendue de la réduction électrolytique, beaucoup moins délicate et plus simple que les anciens procédés électro-métallurgiques par voie humide, où le métal est transporté de l'anode sur la cathode, ou bien est dissous à part par des moyens chimiques et déposé ensuite par le courant.

La couche de matière, soumise à cette réduction, se gonfle jusqu'à tripler de volume, à la fin du traitement. Celui-ci dure cinq jours. pendant lesquels on maintient une intensité de 33 ampères. La masse recueillie est du plomb contenant encore de 8 à $\mathrm{i} 3$ pour I 000 de soufre et les impuretés du minerai; elle est lavée sur une table inclinée et, par lévigation, se séparent ainsi la gangue, les sulfures étrangers. On complète cette purification du métal en l'oxydant au four à réverbère, puis en réduisant la litharge obtenue par le charbon, après lui avoir fait subir, à son tour, un lavage; cette litharge peut, elle-même, être livrée sans autre trans- 
formation à certaines industries : celles de la verrerie, du caoutchouc, par exemple.

Le mélange gazeux d'hydrogène sulfuré, d'hydrogène et d'oxygène qui s'échappe de chaque électrolyseur est aspiré, dans une tuyauterie spéciale en plomb et, par une combustion réglée avec soin, il restitue le soufre qu'il contient; la quantité en varie suivant le degré de transformation où se trouve le sulfure de plomb traité; elle est maxima après les 24 premières heures, puis diminue. On isole ainsi entièrement les parties constitutives de la matière première.

Entre deux cuvettes voisines s'établit, sous l'intensité choisie, une différence de potentiel de 2 volts 9. Quatre piles d'électrolyseurs sont donc reliées en série, les génératrices développant $\mathrm{s} 25$ volts. L'usine possède 220 de ces piles, traitant 10 tonnes de plomb par jour, c'est-à-dire donnant par cheval-heure environ goo grammes de plomb.

L'énergie nécessaire y est apportée des stations hydroélectriques du Niagara, en courant alternatif de 2250 volts, jusqu'à deux moteurs Westinghouse de 300 chevaux ; ceuxci entraînent deux dynamos continues de 250 chevaux, qui maintiennent le voltage nécessaire aux bornes des séries d'électrolyseurs.

Cette métallurgie simplifiée, due à l'esprit d'observation américain, semble, sans doute, ne s'accommoder que de minerais très purs; mais il est possible qu'elle puisse s'adapter, en dehors des galènes, à nombre d'autres sulfures métalliques, et de tous côtés des essais sont faits, depuis l'apparition du procédé Salom, pour en généraliser la méthode. Suivant toute probabilité ils ne resteront pas infructueux et conduiront à de nombreuses innovations dans la métallurgie des minerais sulfurés.

$$
\text { (A suivre) }
$$

P. Pierron,

Chef des travaux

à l'Ecole de Chimie industrielle de Lyon.

\section{JAUGEUR DIFFÉRENTIEL POUR DÉVERSOIR}

Incertitude des jaugeages par déversoirs. - On sait que le débit $Q$ d'un déversoir de longueur. $L$ est donné par la formule :

$$
Q=m \times L . h \sqrt{2 g h}
$$

dans laquelle $m$ est un coefficient empirique qui renferme : le rapport entre l'épaisseur de la nappe déversante sur le seuil et la charge $h$, mesurée entre le seuil et le niveau tranquille à l'amont; les corrections relatives à la contraction latérale, à la forme et à l'épaisseur du seuil s'il n'est pas en mince paroi.

Poncet, Lesbros, Boileau, Hirn, Grœff, Bazin, ont déterminé un certain nombre de valeurs de $m$ sur des déversnirs de longueur, d'épaisseur, de formes et nature différentes, et sous des charges variables. Si l'on fait'des jaugeages en reproduisant exactement leurs conditions d'expérience, on peut utiliser avec chance de précision les coefficients trouvés. Hors de ces conditions, difficilement réalisables en pratique, on n'obtient que des résultats inexacts. Dans tous les cas l'erreur possible dans la mesure de $h$, opération délicate qui suppose un seuil parfaitement horizontal et porte sur une quantité généralement faible - ce qui grandit l'erreur relative - introduit une incertitude dans les résultats.

Ces raisons font qu'on emploie peu les jaugeages par déversoir. Une méthode de mesure précise utilisant ce dispositif présenterait cependant de l'intérêt car le déversoir est d'une installation facile sur les barrages qui existent déjà partout ou des forces sont aménagées, et sur les rivières ou l'on veut créer des chutes on hésiterait moins devant la dépense de ces ouvrages s'ils permettaient d'obtenir avec certitude leurs débits.

Principe du procédé. - Le dispositif suivant, représenté schématiquement fig. I, permet, en faisant déplacer un appareil de mesure devant la nappe déversante, d'en évaluer directement le débit comme le ferait un compteur volumé. trique.

Le principe consiste à isoler entre deux lames minces verticales $\mathbf{a}$ et $\mathbf{b}$ une largeur déterminée e de la nappe déversante, sans en troubler le régime, de telle sorte que la tranche prise est bien celle qui passerait entre deux plans théoriques distants de e. C'est à l'aide de cette tranche élémentaire dont le volume est mesuré par un compteur C ou un enregistreur E, comme on le verra plus loin, que l'on calcule le débit total du déversoir.

Dans le cas théorique d'une nappe régulière, l'appareil étant mis en un point quelconque, si $U$ est le volume qui l'a traversé pendant le temps $T$, le débit à la seconde de la tranche prise de largeur $e$ sera $\frac{U}{T}$ et le débit $Q$ du déver* soir de largeur $L$ :

$$
Q=\frac{L}{e} \times \frac{U}{T}
$$

Cela étant vrai pour une position quelconque de l'appareil, serait encore exact s'il se déplaçait successivement devant tous les points du déversoir en le parcourant pendant ce même temps $T$.

Ce déplacement, indifférent pour connaître le débit d'une nappe théorique, est obligatoire en pratique ou la nappe est irrégulière et la tranche prise e variable d'un point à un autre. Il est donc nécessaire, dans ce cas, de faire déplacer l'appareil d'un mouvement uniforme le long $\mathrm{du}$ déversoir pour qu'il fasse la sommation des débits de tous les points. On comprend, en effet, que restant un même temps devant chaque point la quantité d'eau qu'il reçoit est proportionnelle au débit en ce point et la quantité totale prise sur son parcours devant le déversoir proportionnelle au débit de ce dernier.

D'une façon générale, l'appareil étant en mouvement uniforme le long du déversoir, si $l$ est sa distance du point de départ au bout du temps $t$ et $u$ le volume qui l'a traversé pendant ce temps, $\frac{d u}{d t}$ sera le débit du déversoir au point considéré pour une largeur $e$ de la prise, et $\frac{d l}{e} \times \frac{d t}{d t}$ exprimera le débit du déversoir en ce point pour une longueur $d l$, c'est-à-dire $d Q$.

$$
d Q=\frac{d l}{e} \cdot \frac{d u}{d t}
$$

